International Journal of Algebra, Vol. 8, 2014, no. 18, 889 - 894 HIKARI Ltd, www.m-hikari.com http://dx.doi.org/10.12988/ija.2014.410101

\title{
On Solutions of Indefinite Binary Quadratic Form
}

$$
a u^{2}+b u v+c v^{2}=m
$$

\author{
Bahar Demirtürk Bitim and Ece Özel \\ Sakarya University Department of Mathematics \\ 54187 Sakarya, Turkey
}

Copyright (c) 2014 Bahar Demirtürk Bitim and Ece Özel. This is an open access article distributed under the Creative Commons Attribution License, which permits unrestricted use, distribution, and reproduction in any medium, provided the original work is properly cited.

\begin{abstract}
In this paper, we consider solutions of indefinite binary quadratic forms. Firstly we obtain all integer solutions of the form $u^{2}-5 u v+v^{2}=$ -3 , then we find integer solutions of the most general binary quadratic form $a u^{2}+b u v+c v^{2}=m$ by using the Pell equation $x^{2}-d y^{2}=N$, where $a, b, c \in \mathbb{Z}$ and $m, N$ are nonzero fixed integers.
\end{abstract}

Mathematics Subject Classification: 11E16, 11D09, 11D45, 11J70

Keywords: Binary Quadratic Forms, Continued Fractions, Diophantine Equations, Pell Equations

\section{Introduction}

A binary quadratic form $q(u, v)$ in two variables is defined by

$$
q(u, v)=a u^{2}+b u v+c v^{2},
$$

where $a, b, c \in \mathbb{Z}$. The form can be written simply $q$ or $q=(a, b, c)$.

An integer $m \in \mathbb{Z}$ is represented by $q$ if there are $u, v \in \mathbb{Z}$ with $m=q(u, v)$. The discriminant of $q$ is $d(q)=b^{2}-4 a c$. The form $q$ is positive definite if and only if $d(q)<0$ and $a>0$. The form $q$ is negative definite if and only if $d(q)<0$ and $a<0$. The form $q$ is positive semidefinite if and only if $d(q) \leq 0$ and $(a>0$ or $c>0$ ). The form $q$ is negative semidefinite if and only if $d(q) \leq 0$ 
and $(a<0$ or $c<0)$. The form $q$ is indefinite if and only if $d(q)>0$. For convenience, we will use $d$ instead of $d(q)$ in tide of our paper and we will deal with indefinite quadratic forms. One can consult [5], [6] and [7] for more detailed information about quadratic forms .

Let $d$ be a positive integer which is not a perfect square and $N$ be any nonzero fixed integer. Then the equation $x^{2}-d y^{2}=N$ is known as Pell equation. For $N= \pm 1$, the equations $x^{2}-d y^{2}= \pm 1$ are known as classical Pell equations. If $u^{2}-d v^{2}=N$, we say that $(u, v)$ is a solution of the Pell equation $x^{2}-d y^{2}=N$. We can use $u+v \sqrt{d}$ instead of $(u, v)$ to indicate solutions of the equation $x^{2}-d y^{2}=N$. Among these there is a least positive solution $x_{1}+y_{1} \sqrt{d}$ in which $x_{1}$ and $y_{1}$ have their least positive values. Then the number $x_{1}+y_{1} \sqrt{d}$ is called the fundamental solution of the equation $x^{2}-d y^{2}=N$. See [1], [2], [3] and [4].

The solutions of Pell equations or its more general forms can be easily obtained in terms of the continued fraction of $\sqrt{d}$.

Let $d$ be a positive integer that is not a perfect square. Then there is a continued fraction expansion of $\sqrt{d}$ with period length $l$, such that $\sqrt{d}=$ $\left[a_{0}, a_{1}, a_{2}, \ldots, a_{l-1}, 2 a_{0}\right]$, where

$$
a_{0}=\sqrt{d}, a_{k}=\left\lfloor a_{k}\right\rfloor
$$

and

$$
a_{k+1}=\frac{1}{a_{k}-\left\lfloor a_{k}\right\rfloor}, k=0,1,2,3, \ldots
$$

Recall that $a_{l}=2 a_{0}$ and $a_{l+k}=a_{k}$ for $k \geq 1$. The $n^{\text {th }}$ convergent of $\sqrt{d}$ for $n \geq 0$ is given by

$$
\begin{aligned}
& \frac{p_{n}}{q_{n}}=\left[a_{0}, a_{1}, \ldots, a_{n}\right]=a_{0}+\frac{1}{a_{1}+\frac{1}{a_{2}+\frac{1}{1}}} . \\
& +\frac{1}{a_{n-1}+\frac{1}{a_{n}}}
\end{aligned}
$$

By means of the $n^{\text {th }}$ convergent of $\sqrt{d}$, the fundamental solutions of the equations $x^{2}-d y^{2}= \pm 1$ can be found easily in [7], [9], [10] and [11]. 


\section{Solutions of Binary Quadratic Form $u^{2}-5 u v+$ $v^{2}=-3$}

In [8], Andreescu, Andrica and Cucurezeonu consider the equation $u^{2}-5 u v+$ $v^{2}=-3$. They used Fermat's method of infinite descent for finding the solutions of that equation. In this paper, we firstly deal with the same equation. Indeed, this equation denotes an indefinite binary quadratic form. We use Pell equation $x^{2}-d y^{2}=N$, for finding all solutions of this form.

Let equation $x^{2}-d y^{2}=N$ be solvable and $u+v \sqrt{d}$ be a solution of it. If $x+y \sqrt{d}$ is any solution of the equation $x^{2}-d y^{2}=1$, then $(u+v \sqrt{d})(x+y \sqrt{d})$ is also a solution of $x^{2}-d y^{2}=N$. The solution is said to be associated with the solution $u+v \sqrt{d}$. The set of all solutions associated with each other forms a class of solutions of $x^{2}-d y^{2}=N$. The solution class of the equation $x^{2}-d y^{2}=N$ is denoted by $K$. Further information for the class of solutions of Pell equations is given in [1]. We will give Theorem 2.1, Theorem 2.2 and Theorem 2.3 without proof, since one can find their proofs in [1], [8].

Theorem 2.1. If $u+v \sqrt{d}$ is the fundamental solution of the class $K$ of the equation

$$
x^{2}-d y^{2}=N
$$

and if $x_{1}+y_{1} \sqrt{d}$ is the fundamental solution of equation $x^{2}-d y^{2}=1$, we have the inequalities

$$
\begin{aligned}
& 0 \leqq v \leqq \frac{y_{1}}{\sqrt{2\left(x_{1}+1\right)}} \sqrt{N} \\
& 0<|u| \leqq \sqrt{\frac{1}{2}\left(x_{1}+1\right) N}
\end{aligned}
$$

Theorem 2.2. If $u+v \sqrt{d}$ is the fundamental solution of the class $K$ of the equation

$$
x^{2}-d y^{2}=-N,
$$

and if $x_{1}+y_{1} \sqrt{d}$ is the fundamental solution of equation $x^{2}-d y^{2}=1$, we have the inequalities

$$
\begin{aligned}
& 0<v \leqq \frac{y_{1}}{\sqrt{2\left(x_{1}-1\right)}} \sqrt{N} \\
& 0 \leqq|u| \leqq \sqrt{\frac{1}{2}\left(x_{1}-1\right) N}
\end{aligned}
$$

Theorem 2.3. Let $x+y \sqrt{d}$ be the fundamental solution of the class $K$ of the equation $x^{2}-d y^{2}=N$ and let $x_{1}+y_{1} \sqrt{d}$ be the fundamental solution of the equation $x^{2}-d y^{2}=1$. Then we get all solutions of the class $K$ of the equation $x^{2}-d y^{2}=N$ by $(x+y \sqrt{d})\left(x_{1}+y_{1} \sqrt{d}\right)^{n}$ with $n>0$. 
Lemma 2.4. Let $\left(x_{1}, y_{1}\right)$ be the fundamental solution of the equation $x^{2}-$ $d y^{2}=1$. Then

$$
\left(x_{1}+y_{1} \sqrt{d}\right)^{n}=A_{n}+B_{n} \sqrt{d}
$$

where

$$
A_{n}=\sum_{k=0}^{\left\lfloor\frac{n}{2}\right\rfloor}(d)^{k}\left(\begin{array}{c}
n \\
2 k
\end{array}\right) x_{1}^{n-2 k} y_{1}^{2 k}
$$

and

$$
B_{n}=\sum_{k=0}^{\left\lfloor\frac{n-1}{2}\right\rfloor}(d)^{k}\left(\begin{array}{c}
n \\
2 k+1
\end{array}\right) x_{1}^{n-1-2 k} y_{1}^{2 k+1}
$$

for all $n \in \mathbb{N}$.

Proof. The proof can be seen by the mathematical induction method.

Theorem 2.5. All integer solutions of the binary quadratic form $u^{2}-5 u v+$ $v^{2}=-3$ are given by $(u, v)=\left(4 A_{n}+18 B_{n}, A_{n}+3 B_{n}\right),\left(A_{n}+3 B_{n}, A_{n}-3 B_{n}\right)$, $\left(19 A_{n}+87 B_{n}, 4 A_{n}+18 B_{n}\right)$ and $\left(A_{n}-3 B_{n}, 4 A_{n}-18 B_{n}\right)$ where

$$
A_{n}=\sum_{k=0}^{\left\lfloor\frac{n}{2}\right\rfloor}(21)^{k}\left(\begin{array}{c}
n \\
2 k
\end{array}\right) 55^{n-2 k} 12^{2 k}
$$

and

$$
B_{n}=\sum_{k=0}^{\left\lfloor\frac{n-1}{2}\right\rfloor}(21)^{k}\left(\begin{array}{c}
n \\
2 k+1
\end{array}\right) 55^{n-1-2 k} 12^{2 k+1}
$$

for all $n \in \mathbb{N}$.

Proof. Let us consider the equation $u^{2}-5 u v+v^{2}=-3$. If we multiply both sides of equation by 4 , then we get $(2 u-5 v)^{2}-21 v^{2}=-12$. Now taking $x=2 u-5 v$ and $y=v$ we obtain $x^{2}-21 y^{2}=-12$. Let us determine the fundamental solution of the equation $x^{2}-21 y^{2}=1$, by using the continued fraction expansion of $\sqrt{21}=[4,1,1,2,1,1,8, \ldots]$. Thus it follows that the fundamental solution is $55+12 \sqrt{21}$. By Theorem 2.2 we get $0<$ $v \leqq \frac{12}{\sqrt{2(55-1)}} \sqrt{21}, 0 \leqq|u| \leqq \sqrt{\frac{1}{2}(55-1) 21}$. Therefore we obtain $v=1,2,3,4$ and $u=0,1, \ldots, 17,18$. Then we get four solution classes of the equation $x^{2}-$ $21 y^{2}=-12$. The fundamental solutions of these classes are $3+\sqrt{21},-3+\sqrt{21}$, $18+4 \sqrt{21}$ and $-18+4 \sqrt{21}$. We have all solutions of four classes of the equation 
as $(3+\sqrt{21})(55+12 \sqrt{21})^{n},(-3+\sqrt{21})(55+12 \sqrt{21})^{n},(18+4 \sqrt{21})(55+12 \sqrt{21})^{n}$ and $(-18+4 \sqrt{21})(55+12 \sqrt{21})^{n}$ by Theorem 2.3 . It can be easily seen that $(55+12 \sqrt{21})^{n}=A_{n}+B_{n} \sqrt{21}$ where

$$
A_{n}=\sum_{k=0}^{\left\lfloor\frac{n}{2}\right\rfloor}(21)^{k}\left(\begin{array}{c}
n \\
2 k
\end{array}\right) 55^{n-2 k} 12^{2 k}
$$

and

$$
B_{n}=\sum_{k=0}^{\left\lfloor\frac{n-1}{2}\right\rfloor}(21)^{k}\left(\begin{array}{c}
n \\
2 k+1
\end{array}\right) 55^{n-1-2 k} 12^{2 k+1},
$$

by Lemma 2.4. Thus it follows that all solutions of four classes are given by

$$
\begin{aligned}
(3+\sqrt{21})\left(A_{n}+B_{n} \sqrt{21}\right) & =\left(3 A_{n}+21 B_{n}\right)+\sqrt{21}\left(A_{n}+3 B_{n}\right), \\
(-3+\sqrt{21})\left(A_{n}+B_{n} \sqrt{21}\right) & =\left(-3 A_{n}+21 B_{n}\right)+\sqrt{21}\left(A_{n}-3 B_{n}\right), \\
(18+4 \sqrt{21})\left(A_{n}+B_{n} \sqrt{21}\right) & =\left(18 A_{n}+84 B_{n}\right)+\sqrt{21}\left(4 A_{n}+18 B_{n}\right)
\end{aligned}
$$

and

$$
(-18+4 \sqrt{21})\left(A_{n}+B_{n} \sqrt{21}\right)=\left(-18 A_{n}+84 B_{n}\right)+\sqrt{21}\left(4 A_{n}-18 B_{n}\right) .
$$

Then it is clear that $(u, v)=\left(4 A_{n}+18 B_{n}, A_{n}+3 B_{n}\right),\left(A_{n}+3 B_{n}, A_{n}-3 B_{n}\right)$, $\left(19 A_{n}+87 B_{n}, 4 A_{n}+18 B_{n}\right)$ and $\left(A_{n}-3 B_{n}, 4 A_{n}-18 B_{n}\right)$.

\section{The Solutions of Binary Quadratic Form $a u^{2}+$ $b u v+c v^{2}=m$}

First we look at the case $u^{2}-5 u v+v^{2}=-3$, from this point of view we will obtain all solutions of the indefinite binary quadratic form

$$
a u^{2}+b u v+c v^{2}=m \text {. }
$$

Theorem 3.1. All integer solutions of the indefinite binary quadratic form

$a u^{2}+b u v+c v^{2}=m$ are given by $(u, v)=\left(\frac{(x-b y) A_{n}-(x b-y d) B_{n}}{2 a}, x B_{n}+\right.$ $\left.y A_{n}\right)$.

Proof. Let $(u, v)$ be the solution of the equation (3). By multiplying both sides of (3) with 4 and completing the square we see that

$$
4 a m=(2 a u+b v)^{2}-d v^{2} .
$$

If we take $x=2 a u+b v, y=v$ and $N=4 a m$ in (4), then we obtain $x^{2}-d y^{2}=$ $N$, where $d=b^{2}-4 a c>0$. Now we determine the fundamental solution 
of the equation $x^{2}-d y^{2}=1$, by using the continued fraction expansion of $\sqrt{d}$. Let $x_{1}+y_{1} \sqrt{d}$ be the fundamental solution of the equation $x^{2}-d y^{2}=$ 1. Let $x+y \sqrt{d}$ be the fundamental solution of the class $K$ of the equation $x^{2}-d y^{2}=N$. By Theorem 2.3, we obtain the general solution $x_{n}+y_{n} \sqrt{d}=(x+$ $y \sqrt{d})\left(x_{1}+y_{1} \sqrt{d}\right)^{n}$ of the equation $x^{2}-d y^{2}=N$. Then Lemma 2.4 implies that $\left(x_{1}+y_{1} \sqrt{d}\right)^{n}=A_{n}+B_{n} \sqrt{d}$, with $A_{n}$ and $B_{n}$ given in (1) and (2), respectively. Thus it follows that $x_{n}=x A_{n}+y d B_{n}$ and $y_{n}=x B_{n}+y A_{n}$. Then $(u, v)=$ $\left(\frac{(x-b y) A_{n}-(x b-y d) B_{n}}{2 a}, x B_{n}+y A_{n}\right)$.

\section{References}

[1] T. Nagell, Introduction to number theory, AMS Chelsea Pub, New York, 2010.

[2] E. Landau, Elemantary Number Theory, Chelsea Pub, New York, 1958.

[3] D. Redmond, Number Theory An Introduction, Marcel Dekker, New York, 1996.

[4] M. Jacobson and H. Williams, Solving the Pell Equation, Canadian Math. Soc., Canada, 2009. http://dx.doi.org/10.1007/978-0-387-84923-2

[5] J. Buchmann and U. Vollmer, Binary Quadratic Forms, Springer, 2007. http://dx.doi.org/10.1007/978-3-540-46368-9_2

[6] D.A. Buell, Binary Quadratic Forms Classical Theory and Modern Computations, Springer-Verlag, 1989. http://dx.doi.org/10.1007/978-1-46124542-1

[7] I. Niven, H.S. Zuckerman and H.L. Montgomery, An Introduction to the Theory of Numbers, John Wiley and Suns, Inc., USA, 1991.

[8] T.Andreescu, D. Andrica and I. Cucurezeanu, An Introduction to Diophantine Equations, Birkhauser, 2010. http://dx.doi.org/10.1007/978-08176-4549-6

[9] G.H. Hardy and E.M. Wright, An Introduction to the Theory of Numbers, Oxford Uni. Pres., Oxford, 1979.

[10] K.H. Rosen, Elemantary Number Theory and Its Applications, AddisonWesley Pub. Com., 1986.

[11] W. Bosma and C. Kraaikamp, Continued Fractions, http://www.math.ru.nl/ bosma/Students/CF.pdf

Received: October 21, 2014; Published: December 12, 2014 